\title{
Prevalence of and risk factors for quinolone-resistant Neisseria gonorrhoeae infection in Ontario
}

\author{
Kaede V. Ota MD, Frances Jamieson MD, David N. Fisman MD MPH, Karen E. Jones MD MHSc, \\ Itamar E. Tamari MD, Lai-King Ng PhD, Lynn Towns MLT, Prasad Rawte MS MLT, \\ Alessandro Di Prima BSc, Tom Wong MD MPH, Susan E. Richardson MD
}

$\infty$ See related commentary by Tapsall, page 268

\section{ABSTRACT}

Background: Quinolone-resistant Neisseria gonorrhoeae has swiftly emerged in Canada. We sought to determine its prevalence in the province of Ontario and to investigate risk factors for quinolone-resistant $N$. gonorrhoeae infection in a Canadian setting.

Methods: We used records from the Public Health Laboratory of the Ontario Agency for Health Protection and Promotion in Toronto, Ontario, and the National Microbiology Laboratory in Winnipeg, Manitoba, to generate epidemic curves for $N$. gonorrhoeae infection. We extracted limited demographic data from 2006 quinolone-resistant $N$. gonorrhoeae isolates and from a random sample of quinolonesusceptible isolates. We also extracted minimum inhibitory concentrations for commonly tested antibiotics.

Results: Between 2002 and 2006, the number of N. gonorrhoeae infections detected by culture decreased by $26 \%$ and the number of cases detected by nucleic acid amplification testing increased 6-fold. The proportion of $N$. gonorrhoeae isolates with resistance to quinolones increased from $4 \%$ to $28 \%$ over the same period. Analysis of 695 quinolone-resistant N. gonorrhoeae isolates and 688 quinolone-susceptible control isolates from 2006 showed a higher proportion of men (odds ratio [OR] 3.1, 95\% confidence interval $[\mathrm{CI}] 2.3-4.1)$ and patients over 30 years of age (OR 3.1, 95\% Cl 2.4-3.8) in the quinolone-resistant group. The proportion of men who have sex with men appeared to be relatively similar in both groups (OR 1.4, 95\% Cl 1.1-1.8). Quinolone-resistant strains were more resistant to penicillin $(p<0.001)$, tetracycline $(p<0.001)$ and erythromycin $(p<0.001)$. All isolates were susceptible to cefixime, ceftriaxone, azithromycin and spectinomycin.

Interpretation: During 2006 in Ontario, $28 \%$ of $N$. gonorrhoeae isolates were resistant to quinolones. Infections in heterosexual men appear to have contributed significantly to the quinolone resistance rate. Medical practitioners should be aware of the widespread prevalence of quinolone-resistant $N$. gonorrhoeae and avoid quinolone use for empiric therapy.

Une version française de ce résumé est disponible à l'adresse www.cmaj.ca/cgi/content/full/180/3/287/DC1

CMAJ 2008;180(3):287-90
$\Lambda$ fter declining for a number of years, Neisseria gonorrhoeae infections are once more on the rise in Canada. Between 1997 and 2007, reported incidence of the disease more than doubled, from 15 to 35 cases per $100000 .^{1}$ To address the emergence of quinolone-resistant $N$. gonorrhoeae strains, the empiric treatment regimens for $N$. gonorrhoeae infection were recently revised in the 2006 Canadian Guidelines on Sexually Transmitted Infections. ${ }^{2,3}$ Quinolones are no longer recommended for empiric therapy for $N$. gonorrhoeae infection.

In Canada, quinolone resistance in $N$. gonorrhoeae isolates increased from an estimated $2 \%$ in 2001 to $16 \%$ in $2005 .{ }^{4}$ Demographic risk factors for quinolone-resistant $N$. gonorrhoeae infection have not been studied. American studies have associated quinolone-resistant $N$. gonorrhoeae infection with men who have sex with men, ${ }^{5,6}$ antibiotic use, ${ }^{5,7}$ age above 35 years, ${ }^{5}$ HIV infection ${ }^{5}$ and travel to Asia. ${ }^{6}$ Public health data from the provinces of Quebec ${ }^{8}$ and Alberta ${ }^{2}$ have also suggested an association between quinolone-resistant infection and men who have sex with men. In this study we generated epidemic curves for $N$. gonorrhoeae and quinolone-resistant $N$. gonorrhoeae infection in the province of Ontario. We also investigated demographic risk factors for quinolone-resistant $N$. gonorrhoeae infection.

\section{Methods}

The province of Ontario is the most populous in Canada. Its 12160282 inhabitants account for $38 \%$ of the Canadian population. ${ }^{9}$ The province covers a large geographic area $\left(917741 \mathrm{~km}^{2}\right)$ in the east-central part of the country, with its most densely settled areas in the southern Great Lakes region. The Greater Toronto Area is the most heavily populated metropolitan region in Ontario (population 5555 912). ${ }^{9}$

In this study, we used records for the year 2006 from the Public Health Laboratory of the Ontario Agency for Health Pro-

From The Hospital for Sick Children (Ota, Fisman, Richardson); the University of Toronto (Ota, Jamieson, Fisman, Jones, Richardson); the Public Health Laboratory, Ontario Agency for Health Protection and Promotion, (Jamieson, Fisman, Towns, Rawte, Di Prima, Richardson); the Maple Leaf Medical Clinic (Jones); the Hassle Free Mens Clinic (Tamari), Toronto, Ont.; the Public Health Agency of Canada, National Microbiology Laboratory (Ng), Winnipeg, Man.; and the Public Health Agency of Canada, Community Acquired Infections Division (Wong), Ottawa, Ont. 
tection and Promotion in Toronto, Ontario, and the National Microbiology Laboratory in Winnipeg, Manitoba. The National Microbiology Laboratory is Canada's reference laboratory for the identification and susceptibility testing of $N$. gonorrhoeae. The Public Health Laboratory performs routine susceptibility testing for $N$. gonorrhoeae. It captures virtually all $N$. gonorrhoeae culture isolates from the province of Ontario and performs nucleic acid amplification testing for $N$. gonorrhoeae detection. Other private and academic laboratories in Ontario also perform molecular testing for $N$. gonorrhoeae.

We extracted limited demographic data that included specimen type, testing agency, patient sex and age from all quinolone-resistant isolates of $N$. gonorrhoeae from 2006 in Ontario and from a random sample of quinolone-susceptible $N$. gonorrhoeae isolates. We selected controls using a 1:1 case-to-control ratio with equal frequency from all months.
We classified rectal specimens from men and specimens originating from 2 clinics known to see large numbers of men who have sex with men as those deriving from men who have sex with men. Where patients had multiple gonococcal isolates from a single visit to a clinic, we chose the most resistant isolate for inclusion in our study.

We obtained data from the records of the Public Health Laboratory on penicillinase production and on the minimum inhibitory concentrations of penicillin, ciprofloxacin, tetracycline, spectinomycin, erythromycin, cefixime and ceftriaxone. We extracted data on the minimum inhibitory concentrations of azithromycin from the records of the National Microbiology Laboratory. We designated isolates with resistance to penicillin, tetracycline and erythromycin as "chromosomally mediated resistance in $N$. gonorrhoeae", a marker of multidrug resistance.

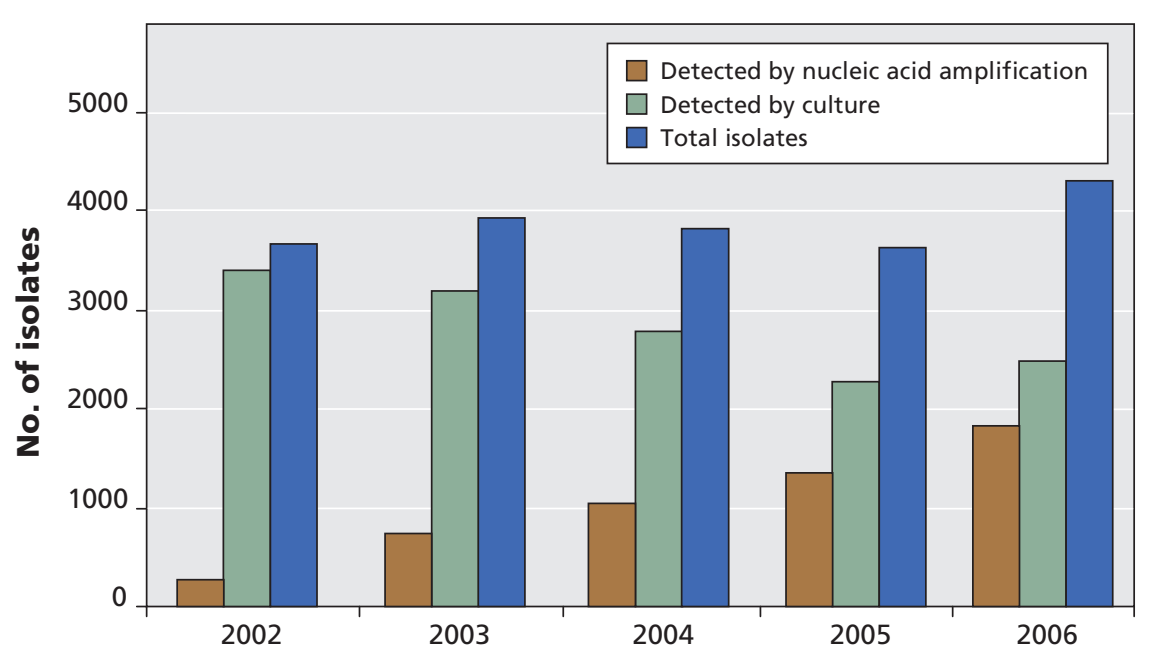

Figure 1: Total number of Neisseria gonorrhoeae isolates detected by culture and by nucleic acid amplification test at the Ontario Public Health Laboratory, 2002-2006.

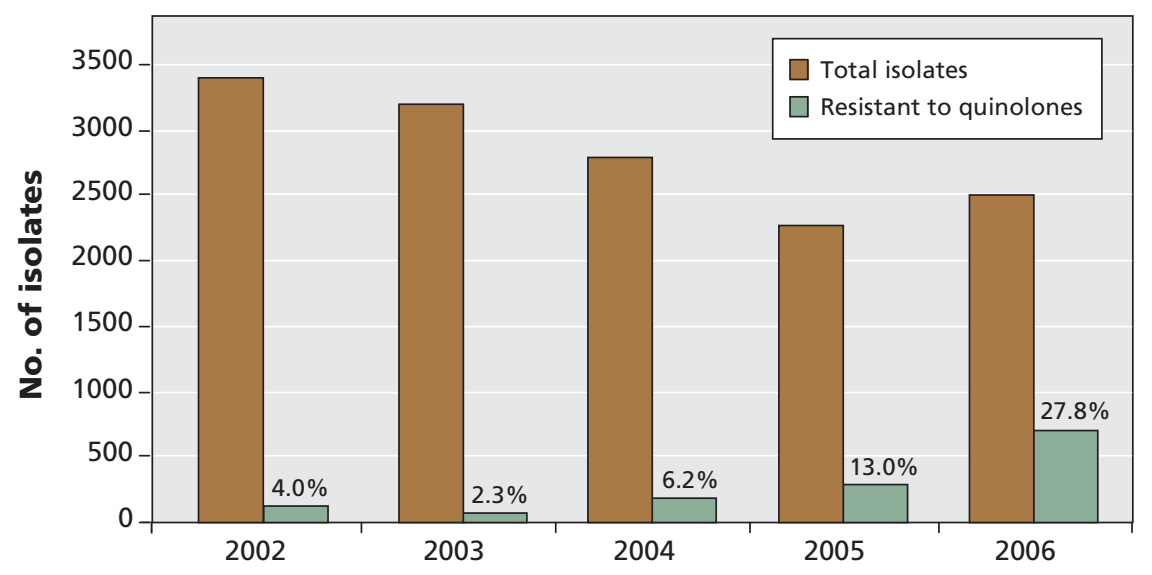

Figure 2: Total number of quinolone-susceptible and quinolone-resistant Neisseria gonorrhoeae isolates in Ontario, 2002-2006.

\section{Results}

The number of N. gonorrhoeae infections diagnosed by the Public Health Laboratory increased over the study period (Figure 1). Between 2002 and 2006, the number of cases of infection detected by culture decreased by 26\% (from 3385 to 2499). The number detected by nucleic acid amplification testing increased nearly 6 -fold (from 303 to 1813). The proportion of $N$. gonorrhoeae isolates with resistance to quinolone increased from $4 \%$ to $28 \%$ over the same period (Figure 2). We noted a rate of quinolone resistance of $55 \%$ at a clinic known to serve men who have sex with men.

We identified 695 quinolone-resistant $N$. gonorrhoeae cases and 688 quinolonesusceptible $N$. gonorrhoeae controls for further analysis. The characteristics of each group are summarized in Table $1 .^{10}$ The quinolone-resistant group had a higher proportion of men $(p<0.001)$ and patients over 30 years of age $(p<0.001)$. Among the men, the proportion who have sex with men was similar in the quinolone-resistant and quinolone-susceptible groups $(p=$ 0.63 ). A multivariable analysis of data restricted to isolates from men showed that having sex with men was not a statistically significant predictor of quinolone resistance after controlling for age above 30 years $(p=0.93)$.

Compared with the culture isolates in the quinolone-susceptible group, the culture isolates in the quinolone-resistant group were more resistant to penicillin $(p<0.001)$, tetracycline $(p<0.001)$ and erythromycin $(p<$ $0.001)$. The latter group were also more likely than the quinolone-susceptible group 
to be multi-drug resistant $(p<0.001)$. All isolates were susceptible to cefixime, ceftriaxone, azithromycin and spectinomycin.

\section{Interpretation}

The prevalence of quinolone-resistant $N$. gonorrhoeae has increased rapidly in Ontario since 2002, rising to 28\% in 2006. The magnitude of the rate of resistance to quinolones is unusually high by any threshold reported in North America. Given Ontario's large population and its status as a major economic centre and national transit hub, its epidemiologic trends are likely to influence trends in other provinces of Canada.

In the United States, the Gonococcal Isolate Surveillance Program is coordinated by the US Centers for Disease Control and Prevention. The program monitors antimicrobial resistance in $N$. gonorrhoeae using data from selected regional laboratories and sexually transmitted infection clinics across the United States. ${ }^{11}$ Resistance to quinolones was reported in $14 \%$ of surveillance isolates in the 2006 annual report of the program. However, closer examination of the data reveals

Table 1: Univariable analysis of characteristics of quinolone-resistant Neisseria gonorrhoeae isolates (cases) and quinolone-susceptible isolates (controls) in Ontario, 2006

\begin{tabular}{|c|c|c|c|c|}
\hline Characteristic & $\begin{array}{c}\text { Quinolone-resistant } \\
\text { isolates, * \% } \\
n=695+\end{array}$ & $\begin{array}{l}\text { Quinolone-susceptible } \\
\text { isolates, }{ }^{*} \% \\
n=688\end{array}$ & Odds ratio $(95 \% \mathrm{Cl})$ & $p$ value \\
\hline \multicolumn{5}{|l|}{ Patient sex } \\
\hline Male & 89.4 & 73.2 & $3.1(2.3-4.1)$ & $<0.001$ \\
\hline Female & 10.6 & 26.8 & & \\
\hline Patient age, yr & & & $3.1(2.4-3.8)+\dagger$ & $<0.001$ \\
\hline$<20$ & 3.2 & 7.3 & & \\
\hline $20-29$ & 29.6 & 52.6 & & \\
\hline 30-39 & 30.4 & 25.4 & & \\
\hline $40-49$ & 25.3 & 11.2 & & \\
\hline$\geq 50$ & 11.5 & 3.5 & & \\
\hline \multicolumn{5}{|l|}{$\begin{array}{l}\text { Patient is man who has sex } \\
\text { with men }\end{array}$} \\
\hline Yes & 29.9 & 23.6 & $1.4(1.1-1.8)$ & 0.63 \\
\hline No & 70.1 & 76.4 & & \\
\hline \multicolumn{5}{|l|}{ Antibiotic susceptibility } \\
\hline \multicolumn{5}{|l|}{ Penicillin $¥$} \\
\hline Resistant or intermediate & 98.4 & 89.4 & $7.4(3.9-14.0)$ & $<0.001$ \\
\hline Susceptible & 1.6 & 10.6 & & \\
\hline $\begin{array}{l}\text { Penicillinase-producing } \\
\text { N. gonorrhoeae }\end{array}$ & 3.4 & 3.5 & $1.0(0.6-1.8)$ & 0.97 \\
\hline \multicolumn{5}{|l|}{ Tetracycline§ } \\
\hline Resistant or intermediate & 98.0 & 81.1 & $11.3(6.4-19.9)$ & $<0.001$ \\
\hline Susceptible & 2.0 & 18.9 & & \\
\hline \multicolumn{5}{|l|}{ Erythromycinף } \\
\hline Resistant or intermediate & 66.2 & 14.8 & $11.2(8.6-14.6)$ & $<0.001$ \\
\hline Susceptible & 33.8 & 85.2 & & \\
\hline \multicolumn{5}{|l|}{ Multidrug resistant** } \\
\hline Yes & 19.7 & 1.2 & $20.9(10.1-42.9)$ & $<0.001$ \\
\hline No & 80.3 & 98.8 & & \\
\hline
\end{tabular}

The minimum inhibitory concentration breakpoints of the Clinical and Laboratory Standards Institute (CLSI) were used to determine antibiotic susceptibility status for all antibiotics except erythromycin.

*The CLSI minimum inhibitory concentration breakpoints for ciprofloxacin were as follows: susceptible $=\leq 0.06 \mu \mathrm{g} / \mathrm{mL}$, intermediate $=0.12-0.5 \mu \mathrm{g} / \mathrm{mL}$ and resistant $=\geq 1 \mu \mathrm{g} / \mathrm{mL}$.

tOne of the isolates had a minimum inhibitory concentration of $0.5 \mu \mathrm{g} / \mathrm{mL}$ (intermediate).

$\ddagger$ The CLSI minimum inhibitory concentration breakpoints for penicillin were as follows: susceptible $=\leq 0.06 \mu \mathrm{g} / \mathrm{mL}$, intermediate $=0.12-1.0 \mu \mathrm{g} / \mathrm{mL}$, resistant $=\geq 2 \mu \mathrm{g} / \mathrm{mL}$.

$\S$ The CLSI minimum inhibitory concentration breakpoints for tetracycline were as follows: susceptible $=\leq 0.25 \mu \mathrm{g} / \mathrm{mL}$, intermediate $=0.5-1.0 \mu \mathrm{g} / \mathrm{mL}$, resistant $=\geq 2 \mu \mathrm{g} / \mathrm{mL}$

१The minimum inhibitory concentration breakpoint for erythromycin resistance was $\geq 2 \mu \mathrm{g} / \mathrm{mL}$. $^{10}$

**Chromosomally mediated resistance in $N$. gonorrhoeae.

††For age above 30 years. 
wide regional variations. Some west coast regions reported quinolone resistance in as many as $35 \%-45 \%$ of isolates, whereas northeastern and southern surveillance sites reported rates lower than $10 \% .{ }^{11} \mathrm{~N}$. gonorrhoeae isolates in Ontario appear to be approaching the levels of quinolone-resistance that are seen in west coast areas of the United States.

The Gonococcal Isolate Surveillance Program reported that $39 \%$ of isolates from men who have sex with men were resistant to quinolone. ${ }^{11}$ Our study found resistance in $55 \%$ of the isolates that originated from a clinic known to serve large numbers of men who have sex with men. To our knowledge, this rate of resistance has not been reported for any population in North America to date.

Our findings reinforce the current recommendations in Canada that quinolones not be used for empiric therapy for $N$. gonorrhoeae infection. ${ }^{3}$ The use of ciprofloxacin and ofloxacin should be limited to instances in which susceptibility to quinolone has been proven. ${ }^{3}$ Anecdotally, the use of quinolones for the empiric treatment of $N$. gonorrhoeae infection appears to persist in the community, perhaps because of the convenience, safety and tolerability of quinolones.

All of the isolates in our study were susceptible to cefixime and ceftriaxone, which indicates that these drugs remain suitable for empiric therapy in Canada. However, isolates with reduced susceptibility to third-generation cephalosporins have been reported in $\mathrm{Asia}^{12,13}$ and the United States. ${ }^{11}$ In people with an allergy to $\beta$-lactam, a single oral dose of $2 \mathrm{~g}$ of azithromycin or a single oral dose of $2 \mathrm{~g}$ of spectinomycin administered intramuscularly are options. ${ }^{3}$ Unfortunately, the azithromycin regimen has been associated with significant gastrointestinal side effects, ${ }^{3}$ and resistance to azithromycin has been reported in the United States. ${ }^{11}$ Access to spectinomycin is limited to the Health Canada Special Access Program and is not suitable for treatment of pharyngeal $N$. gonorrhoeae infection. ${ }^{3}$ The significant rates of resistance to penicillin, tetracycline and erythromycin in our study preclude their empiric use in Ontario.

Continued testing for antibiotic susceptibility is necessary to ensure that $N$. gonorrhoeae infections with multi-drug resistance are quickly identified and contained. ${ }^{14}$ This will be a challenge because the use of urine specimens for molecular testing, in place of more invasive urethral and endocervical specimens, has been gaining popularity. Molecular testing is rapidly replacing conventional culture, which is required for susceptibility testing.

\section{Limitations}

We note that the reported number of tests performed annually in Ontario using nucleic acid amplification may be an underestimate. It is likely to be even higher than that presented in Figure 1 , because molecular testing is also performed at private and academic laboratories. Unfortunately, the data needed to estimate the volume and impact of those tests are not currently available.

\section{Conclusions}

The Public Health Agency of Canada continues to recommend the use of culture testing over tests using nucleic acid amplification in an effort to maintain susceptibility testing for both patient management and surveillance. ${ }^{3}$ The importance of using culture testing for $N$. gonorrhoeae infection needs to be communicated to clinicians, laboratories and public health organizations.

Currently at the national level, no formal surveillance program is in place to collect and compare data on the rates of quinolone-resistant $N$. gonorrhoeae infection across Canada. Ultimately, a national sentinel surveillance system will be necessary to ensure that susceptibility testing continues and that antimicrobial susceptibility in N. gonorrhoeae is monitored. ${ }^{14}$

This article has been peer reviewed.

Competing interests: None declared.

Contributors: Kaede Ota, Frances Jamieson, David Fisman, Karen Jones, Itamar Tamari and Susan Richardson designed the study. Kaede Ota, LaiKing Ng, Lynn Towns, Prasad Rawte, Alessandro Di Prima and Tom Wong acquired the data. Statistical analysis was performed by Kaede Ota and David Fisman. All of the authors participated in drafting or revising the manuscript and approved the final version to be published.

Funding: No external funding was received for this research.

\section{REFERENCES}

1. Public Health Agency of Canada. Reported cases of notifiable STI from January to September 30, 2006 and January 1 to September 30, 2007 and corresponding annual rates for 2006 and 2007. Ottawa (ON): The Agency; 2008. Available: www.phac-aspc.gc.ca/std-mts/stdcases-casmts/chlm-08-eng.php (accessed 2008 Oct. 9)

2. Public Health Agency of Canada. Canadian guidelines on sexually transmitted infections. Ottawa (ON): The Agency; 2006.

3. Public Health Agency of Canada. Gonococcal infections. Ottawa (ON): The Agency; 2008. Available: www.phac-aspc.gc.ca/std-mts/sti_2006/pdf/506_Gonococcal_ Infections.pdf (accessed 2008 Sept. 8).

4. Public Health Agency of Canada. Epi Update: Ciprofloxacin resistance to Neisseria gonorrhoeae in Canada, 2006. Ottawa (ON): The Agency; 2007. Available: www.phac-aspc.gc.ca/sti-its-surv-epi/ciprofloxacin_e.html (accessed 2008 Sept. 8).

5. Bauer HM, Mark KE, Samuel M, et al. Prevalence of and associated risk factors for fluoroquinolone-resistant Neisseria gonorrhoeae in California, 2000-2003. Clin Infect Dis 2005;41:795-803.

6. US Centers for Disease Control and Prevention. Increases in fluoroquinolone-resistant Neisseria gonorrhoeae among men who have sex with men - United States, 2003, and revised recommendatons for gonorrhea treatment, 2004. MMWR Morb Mortal Wkly Rep 2004;53:335-8.

7. Iverson CJ, Wang SA, Lee MV, et al. Fluoroquinolone resistance among Neisseria gonorrhoeae isolates in Hawaii, 1990-2000. Sex Transm Dis 2004:31:702-8.

8. Augmentation du nombre de souches de Neisseria gonorrhoeae résistantes aux fluoroquinolones au Québec. Québec (QC): Santé et Services sociaux, Gouvernement du Québec. Available: http://publications.msss.gouv.qc.ca/acrobat/f/documentation 12007/07-342-01.pdf (accessed 2008 Sept. 8).

9. Population and dwelling counts, for Canada, provinces and territories, census divisions, and census subdivisions (municipalities), 2006 and 2001 censuses $-100 \%$ data. Ottawa (ON): Statistics Canada; 2007. Available: www12.statcan.ca/english /census06/data/popdwell/Table.cfm?T=304\&SR=1\&S=10\&O=D\&RPP=10\&PR=35 (accessed 2008 Oct. 9).

10. Ehret JM, Nims LJ, Judson FN. A clinical isolate of Neisseria gonorrhoeae with in vitro resistance to erythromycin and decreased susceptibility to azithromycin. Sex Transm Dis 1996;23:270-2.

11. Department of Health and Human Services, US Centers for Disease Control and Prevention. Sexually transmitted disease surveillance 2006 supplement. Gonococcal Isolate Surveillance Project (GISP) annual report 2006. Atlanta (GA): The Department; 2008. Available: www.cdc.gov/std/gisp2006/GISPSurvSupp 2006Short.pdf (accessed 2008 Oct. 9).

12. Ye S, Su X, Wang Q, et al. Surveillance of antibiotic resistance of Neisseria gon orrhoeae isolates in China, 1993-1998. Sex Transm Dis 2002;29:242-5.

13. Yokoi S, Deguchi T, Ozawa T, et al. Threat to cefixime treatment for gonorrhea Emerg Infect Dis 2007;13:1275-7.

14. Mann J, Kropp R, Wong T, et al. Gonorrhea treatment guidelines in Canada: 2004 update. CMAJ 2004;171:1345-6.

Correspondence to: Dr. Susan E. Richardson, Division of Microbiology, Pediatric Laboratory Medicine, The Hospital for Sick Children, 555 University Ave., Toronto ON M5G 1X8; fax416 813-6257; susan.richardson@sickkids.ca 